\title{
Effet des acides gras sur l'inflammation et le cancer
}

\author{
Jean-Marc BLOUIN ${ }^{1}$ \\ Valeria E. CHAVES ${ }^{1,2}$ \\ Sylvie BORTOLI ${ }^{1}$ \\ Claude FOREST ${ }^{1}$ \\ ${ }^{1}$ Inserm UMR-S 747 ; Université Paris Descartes, \\ Centre Universitaire, 45 rue des Saints-Pères, \\ 75006 Paris Fax : 0142863868 \\ <claude.forest@univ-paris5.fr> \\ ${ }^{2}$ Department of Physiology, \\ School of Medicine of Ribeirão Preto, \\ Avenida Bandeirantes 3900, 14.049 Ribeirão \\ Preto, S.P. Brésil
}

Le processus inflammatoire inclut la libération de cytokines pro-inflammatoires, de prostaglandines et la formation d'espèces réactives de l'oxygène (ERO) qui combattent les pathogènes, virus et bactéries, attaquant l'organisme. Bien que ce processus soit, en règle générale, transitoire et suivi par une réaction anti-inflammatoire, il arrive que l'organisme maintienne une production élevée de ces cytokines, ce qui peut conduire à une situation d'inflammation chronique et favoriser l'émergence d'un processus tumoral [1]. Un exemple récent illustre très bien cette relation. Lorsque le gène pro-inflammatoire codant la kinase I-Kappa-B (IKK beta) est invalidé chez la souris, I'incidence de cancer et la croissance de tumeurs est réduite de $80 \%$ [2].

Une meilleure connaissance du mécanisme inflammatoire ainsi que des agents favorisant ou à l'inverse inhibant le processus devrait permettre d'éviter ou de contrôler les effets secondaires. Parmi les substances naturelles agissant sur l'inflammation, les acides gras (AG) ont une place à part. II est en effet connu depuis longtemps que les eicosanoïdes (prostaglandines$P G$, leukotriènes- $L T$, tromboxanes- $T X$ et acides hydroxyeicosatetraenoïques-HETE) sont des médiateurs de la réponse inflammatoire. Or, les eicosanoïdes sont engendrés par les AG polyinsaturés (AGPI) à 20 carbones, notamment I'acide arachidonique (C20:4 omega-6), libérés des phospholipides membranaires, principalement grâce à l'action de la phospholipase A2. Les AG ainsi libérés sont substrats des enzymes de synthèse des eicosanoïdes. Les AGPI de la

\begin{abstract}
A substantial body of evidence supports the conclusion that chronic inflammation is a causative factor in a variety of cancers. Inflammatory mediators include metabolites of arachidonic acid, cytokines, chemokines, and free radicals. These mediators increase cell proliferation, mutagenesis, oncogene activation, with ultimately the loss of cell growth control. Among the nutritional factors that can influence inflammation and cancerogenesis, fatty acids are clearly the most directly involved. Indeed, omega-3 and omega-6 polyunsaturated fatty acids exert opposite effects on inflammation and potentially on tumor formation. The present review analyses in a non-exhaustive manner some of the mechanisms that have been proposed to explain how omega- 6 can be detrimental while omega- 3 have beneficial effects.
\end{abstract}

Key words: fatty acids, inflammation, cancer, prostaglandis, COX-2, omaga-3

famille omega-6, dont fait partie l'acide arachidonique, et ceux de la famille omega- 3 sont issus respectivement des acides linoléique (C18:2 omega-6) et linolénique (C18:3 omega-3) par désaturation et élongation. Les acides linoléique et linolénique ne peuvent être synthétisés par l'organisme. En revanche, ils sont produits dans le règne végétal. Ils doivent donc être apportés à l'organisme par I'alimentation et sont, à ce titre, appelés " essentiels » car nécessaires à la vie. Par conséquent, la variation de la qualité des AG alimentaires ingérés sous la forme de triglycérides (triacylglycerol) des huiles alimentaires végétales doit influer sur la composition des phospholipides et entrainer des réponses inflammatoires différentielles. Par ailleurs, plusieurs études montrent que les AGPI omega- 6 favorisent le développement tumoral, tandis que les omega-3 ont un effet protecteur $[3,4]$.

\section{Les acides gras alimentaires et leur devenir cellulaire}

Dans les huiles alimentaires, les AG sont estérifiés en triglycérides. Les huiles de poissons sont riches en AGPI omega-3, alors que I'huile de palme est constituée principalement d'AG saturés et l'huile d'olive contient en particulier l'oléate, un AG mono-insaturé de la série omega-9. À l'état post-prandial, à la suite de leur absorption intestinale, les AG sont véhiculés dans le sang par les chylomicrons (figure $1 A$ ). On les retrouve aussi sous forme de lipoprotéines issues du foie. La lipoprotéine lipase synthétisée et sécrétée majoritairement par le tissu adipeux, hydrolyse ces triglycérides à la surface luminale des cellules endothéliales capillaires et les AG ainsi libérés sont captés par les tissus (figure 1A). À l'inverse, en période de jeûne, les AG du sang proviennent essentiellement du tissu adipeux, par un mécanisme de lipolyse des triglycérides de stockage (figure 1B). Dans le plasma, les AG non-estérifiés (AGNE) sont véhiculés par l'albumine, la fraction minoritaire non liée à l'albumine étant celle des «AG libres » $(A G L)$, souvent confondue avec les AGNE. Les $A G L$ pénètrent dans les cellules à l'aide d'une protéine transmembranaire dénommée «translocateur des $\mathrm{AG}$ » (FAT) et peuvent s'échanger avec les AG des phospholipides de la membrane plasmique pour en modifier, ou interférer avec, un signal issu de la membrane (figure 2). Par ailleurs, les tissus lipogéniques tels que le foie et, tout au moins chez les rongeurs, le tissu adipeux, mais aussi les tissus tumoraux, ont la capacité de synthétiser de novo les AGNE à partir du glucose. Dans la cellule, les AGNE sont pris en charge par une protéine cytoplasmique de liaison des lipides (FABP). Ils peuvent subir une série de modifications, et leur activation en acyl-CoA constitue un préalable à certaines de ces modifications: estérification, oxydation mitochondriale ; ils peuvent également entrer dans des réactions d'élongation, de désaturation, d'oxydation par les peroxysomes ou les microsomes ou bien être peroxydés, comme dans le cas des AGPI (figure 3). Ces différentes étapes ont été schématisées dans un article de 

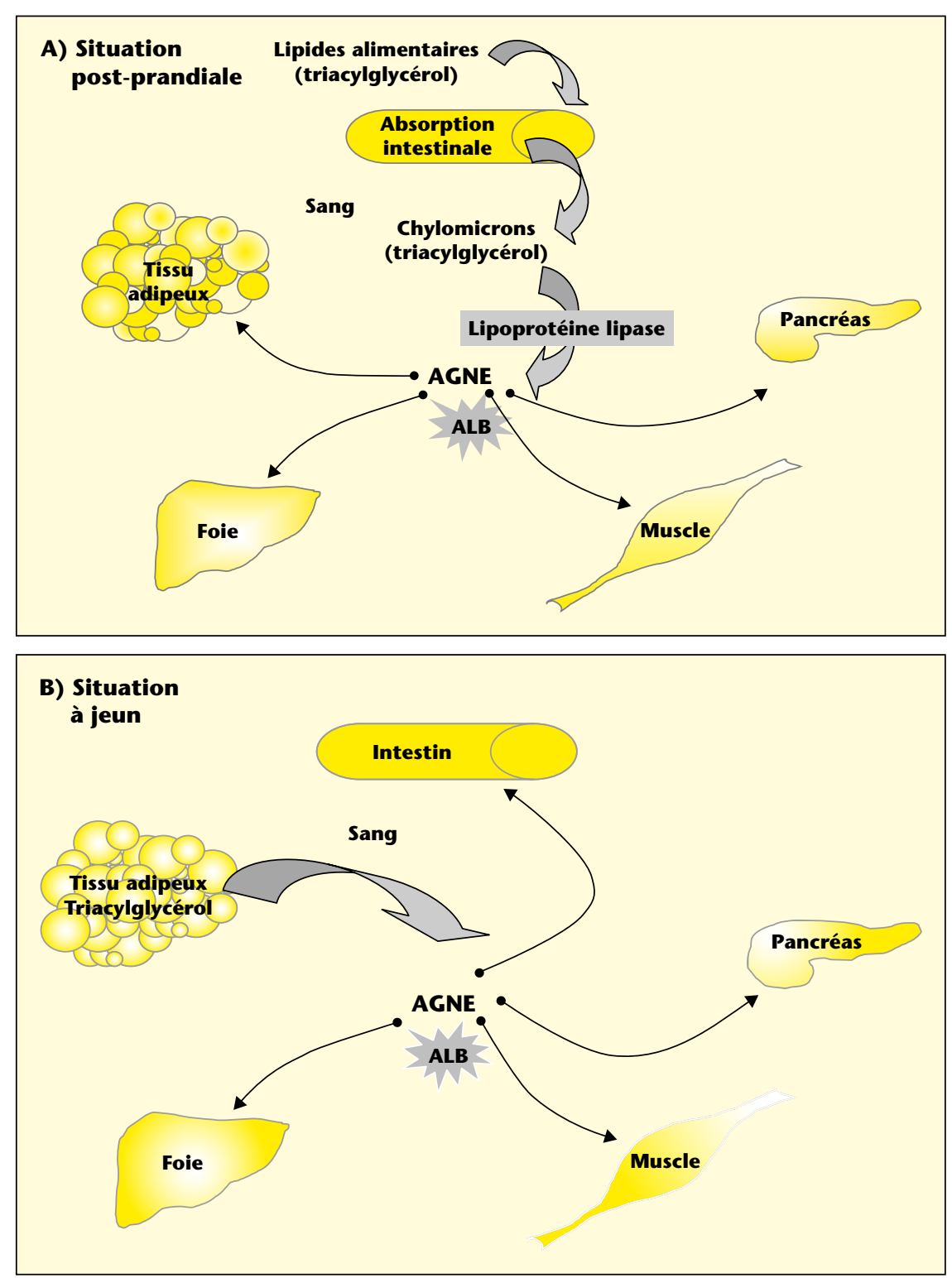

Figure 1. Origine des acides gras du sang. A) En situation post-prandiale (après un repas), les AG non estérifiés (AGNE) du sang proviennent des chylomicrons, issus de l'absorption intestinale des lipides alimentaires, grâce à I'action de la lipoprotéine lipase. La majeure partie des AGNE est liée à l'albumine (ALB). Les AG libres servent alors de substrat énergétique aux tissus. B) À jeun, les AGNE du sang proviennent presque exclusivement des triglycérides (triacylglycérol) du tissu adipeux par lipolyse.

revue récent [4]. La synthèse des eicosanoïdes se faisant à partir de l'acide arachidonique issu des phospholipides membranaires, l'étude de I'incorporation des AGPI dans ces phospholipides revêt une importance particulière.

\section{Acides gras, phospholipides et mécanismes membranaires}

Les phospholipides des membranes cellulaires peuvent être soit néosynthétisés soit subir une modification de leur composition en AG par remodelage. À la base de ce dernier méca- ailleurs, la position libre du lysophospholipide est réacylée par un nouvel $A G$, différent du premier, sous l'action de l'acyl transférase. Ainsi, l'apport nutritionnel joue un rôle déterminant sur la composition des phospholipides en AG.

La qualité des AG des phospholipides des membranes influence profondément leur fluidité et la relation de ces phospholipides avec les protéines de ces membranes. En effet, un grand nombre de protéines sont ancrées dans les membranes par un résidu acyl lié à leur extrémité $\mathrm{N}$-terminale. La qualité de l'AG lié à la protéine peut moduler son activité. Par exemple, I'enrichissement en acide docosahexaénoïque (DHA, C22:6 omega-3), un AG présent en grande quantité dans les huiles de poisson et indispensable au développement de l'organisme, en particulier du cerveau, augmente la sensibilité du muscle cardiaque à une stimulation adrénergique, vraisemblablement par une augmentation de l'activité de l'adénylyl cyclase [5].

\section{Régulation de la synthèse des prostaglandines et inflammation}

Les cyclo-oxygénases (COX), ou prostaglandine $\mathrm{H}$ synthases, sont des enzymes clés de la biosynthèse des prostanoïdes - prostaglandines et thromboxanes (figure 3). De nombreuses observations suggèrent que les $\mathrm{COX}$, en particulier l'isoforme 2 (COX-2) et certaines prostaglandines comme PGE-2 sont impliquées dans la cancérogenèse [6]. D'une part, une forte expression de COX-2 a été décrite dans de nombreuses lignées de cellules tumorales coliques et, chez l'homme, une surexpression de COX-2 est observée dans environ $80 \%$ des cancers colorectaux ainsi que dans $40 \%$ à $50 \%$ des polypes adénomateux, à un stade relativement précoce de la cancérogenèse [7]. D'autre part, les prostaglandines peuvent stimuler la prolifération cellulaire, induire la sécrétion de cytokines, diminuer les défenses immunitaires et inhiber l'apoptose des cellules tumorales [8]. Enfin, les anti-inflammatoires non stéroïdiens (AINS) comme l'aspirine, inhibiteurs de COX-2, réduisent I'incidence de cancérogenèse colique chez la souris [9]. La transfection de cellules épithéliales intestinales de rat avec le gène COX-2 leur confère un phénotype tumoral associé à une augmentation de capacité d'adhérence des cellules et à une diminution de la sensibilité à des agents proapoptotiques [10]. Lors d'une agression de l'organisme, les macrophages colonisant les tissus induisent COX-2 et produisent de grandes quantités de PGE-2 et de PGF2 $\alpha$. De plus, PGE-2 est capable d'induire COX-2, ce qui revient à augmenter sa propre production, et 


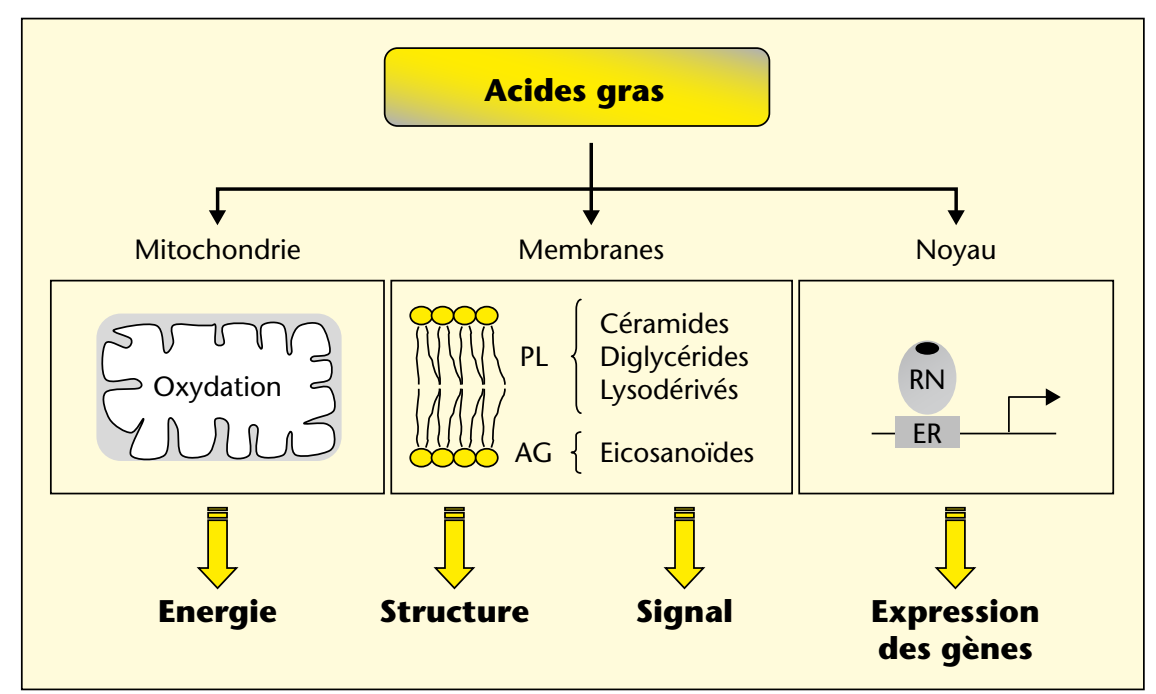

Figure 2. Mécanismes d'action intracellulaire des acides gras. Les AG peuvent être $\beta$-oxydés mais sont aussi échangés avec les $A G$ des phospholipides (PL) de membranes. Ils jouent donc un rôle de structure, pouvant par exemple moduler la fluidité membranaire. L'hydrolyse des phospholipides donne naissance à des molécules de signalisation (céramides, diglycérides, lysodérivés) et aux eicosanoïes. Les AG sont aussi des ligands de récepteurs nucléaires (RN) susceptibles de se lier à des éléments de réponse (ER) dans le promoteur de gènes cibles pour en moduler l'activité et donc l'expression de ces gènes.

entraîne une stimulation de l'interleukine 6 (IL-6) par les macrophages [11]. L'IL-6, comme le facteur de nécrose tumorale alpha (TNF- $\alpha$ ), est une cytokine à forte activité inflammatoire. Il est donc tentant de conclure ici qu'un apport alimentaire riche en AGPI omega-6, est favorable à la synthèse d'acide arachidonique, qui libérée des phospholipides de membranes par la phospholipase A2 sert de substrat à COX-2 pour la formation de prostaglandines pro- inflammatoires, elles-mêmes potentiellement cancérogènes (figure 4).

Un enrichissement des membranes en AGP omega-3, tels que le DHA et l'acide eicosapentaenoïque (EPA), réduit la proportion d'acide arachidonique, entraînant une diminution de la production de PGE-2. Ces AGPI inhibent aussi la formation du TNF- $\alpha$ et de plusieurs interleukines par les monocytes, les macrophages ou les cellules endothéliales. Par ailleurs,

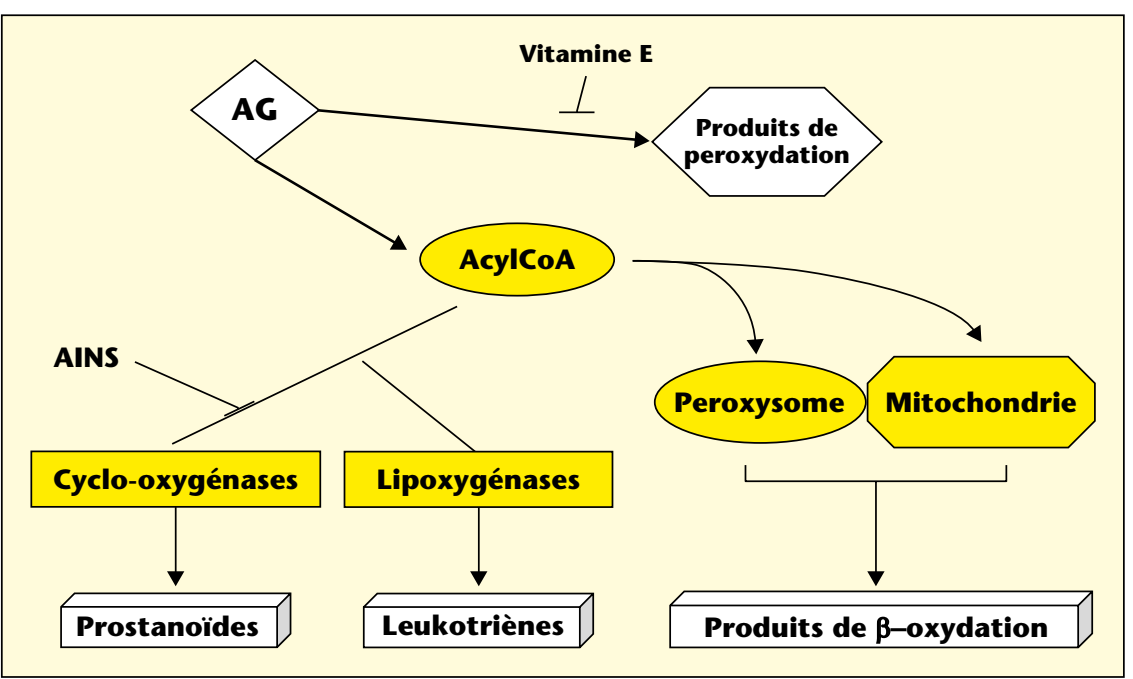

Figure 3. Les acides gras comme molécules de signalisation. Les AG peuvent être peroxydés. Les antioxydants comme la vitamine E inhibent ce processus. Leur activation en acyl-COA les entraîne vers l'oxydation mitochondriale ou peroxysomale, en fonction de leur longueur de chaîne et de leur degré d'insaturation pour donner naissance à des produits intermédiaires issus de cette $\beta$-oxydation. Ils peuvent aussi être pris en charge par les cyclooxygénases ou les lipoxygénases, entraînant la formation de prostanoïdes ou de leukotriènes. Les anti-inflammatoires non stéroidiens (AINS) inhibent l'activité cyclo-oxygénase, réduisant la production de prostanoïdes.
I'EPA est un substrat pour les COX et les lipoxygénases (LOX) conduisant ainsi à la synthèse de PG alternatives non-inflammatoires [6]. De récentes études ont permis l'identification d'un groupe nouveau de médiateurs lipidiques, les resolvines-E et resolvines- $D$, produites respectivement à partir d'EPA et de DHA [6] (figure 4). Ces resolvines exercent une action de réduction de l'inflammation, d'où leur nom anglais de resolvins. Ainsi, un régime alimentaire riche en huiles de poisson, donc en omega-3, semble donc plutôt anti-inflammatoire et potentiellement anti-cancérogène.

\section{Peroxydation des acides gras, inflammation et cancer}

Les produits de peroxydation des AG dépendent des doubles liaisons. Les AG omega-3 sont plus sensibles à l'oxydation que les $A G$ omega-6. Des dommages à l'ADN peuvent être induits par ces produits peroxydés essentiellement en relation avec la formation initiale de radicaux libres, qui possèdent un électron célibataire entraînant leur réactivité avec différentes molécules. Parmi tous les composés synthétisés, les plus réactifs sont les peroxyls, les hydroxyls, à côté desquels on trouvera aussi les anions radicalaires de type superoxyde et le monoxyde d'azote. Ces derniers sont, à l'origine, peu réactifs mais peuvent servir de précurseurs à des dérivés beaucoup plus actifs [12]. Les radicaux libres proviennent du catabolisme des AG au niveau de la chaîne respiratoire. Si la production de radicaux libres est trop importante pour être prise en charge totalement par les systèmes de défense antioxydante (vitamines $\mathrm{C}$ et $\mathrm{E}$, caroténoïdes et systèmes enzymatiques), un stress oxydant se développe, entraînant des lésions cellulaires, notamment la formation d'adduits à l'ADN potentiellement générateurs de mutations. Les dommages à I'ADN dus aux radicaux libres entraînent des changements de l'expression de différents gènes et une dérégulation du cycle cellulaire pouvant augmenter le développement de cellules cancéreuses. À l'inverse, le phénomène d'apoptose lié à l'apparition d'un stress oxydant peut entraîner la destruction de cellules en prolifération active. II est clair que les radicaux libres sont augmentés en cas d'inflammation mais la liaison de ce phénomène avec la cancérogenèse n'est pas complètement établie. Toutefois, l'implication différentielle des AG saturés et des AGPI dans l'incidence des cancers peut en partie s'expliquer par des capacités différentes de peroxydation. Toutefois, il n'existe pas, à ce jour, d'argument expérimental montrant que les AGPI omega-3 influencent le processus cancérigène en modifiant la production de radicaux libres chez I'homme. 


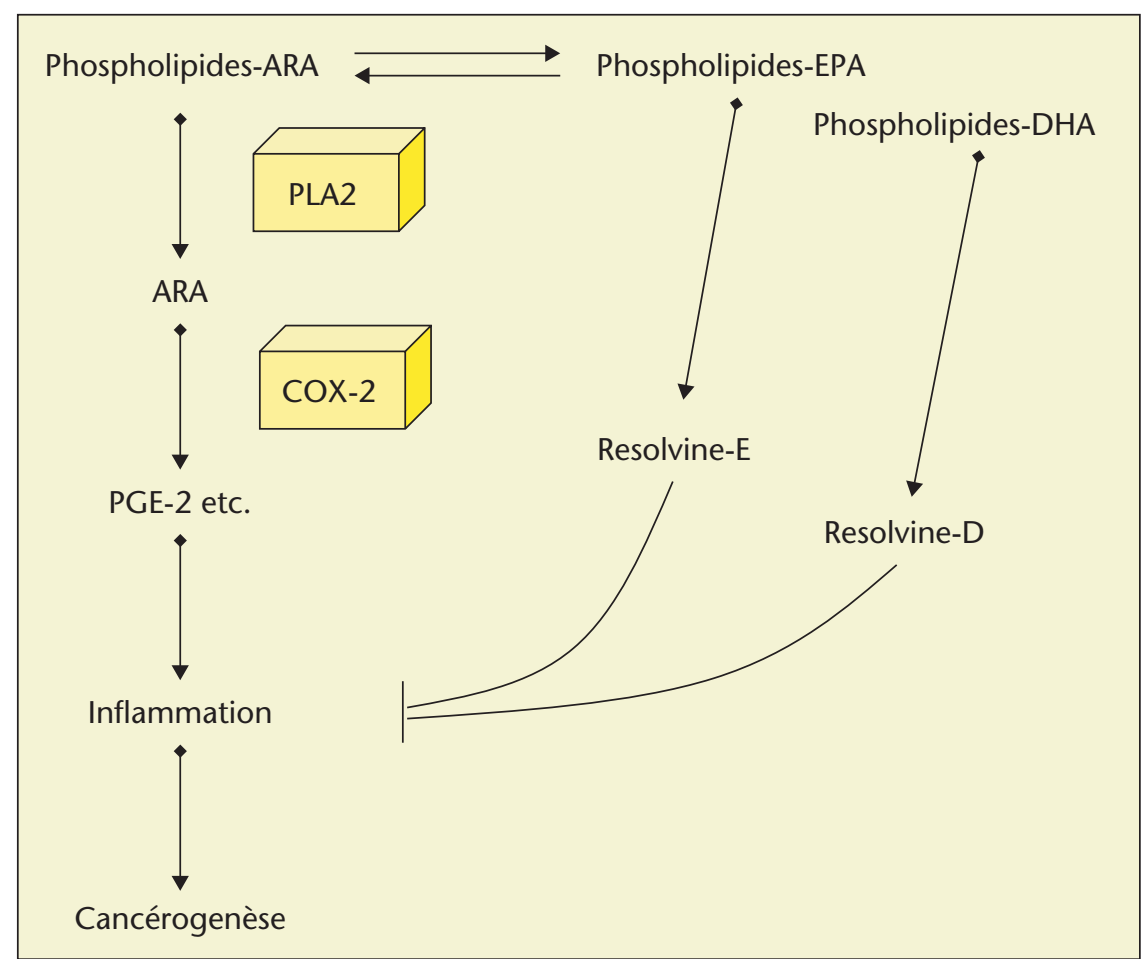

Figure 4. Lien entre phospholipides, inflammation et cancer. Les phospholipides enrichis en acide arachidonique (ARA) précurseur des prostaglandines, notamment de PGE-2 pro-inflammatoire favorisent l'inflammation qui, si elle devient chronique, peut concourir à I'apparition de cellules cancéreuses. La phospholipase A2 (PLA2) est responsable de l'hydrolyse des phospholipides membranaires, alors que la COX-2 utilise I'ARA pour la production de prostaglandines. L'ARA des phospholipides peut s'échanger avec d'autres AG comme les omega-3 (EPA) ou (DHA) qui ne donnent pas naissance aux prostaglandines pro-inflammatoires et, par compétition, la production de ces dernières en est réduite. Les phospholipides-EPA ou -DHA peuvent aussi donner naissance à des résolvines qui présentent une activité anti-inflammatoire.

\section{Régulation de l'expression des gènes par les acides gras et leurs dérivés}

Il est admis que les gènes dont l'expression est modulée par les AG codent des protéines impliquées dans le transport ou le métabolisme de ces AG. L'étude des mécanismes de régulation a fait l'objet de nombreux travaux mais aucune règle générale n'a pu encore être établie [13]. De multiples études ont eu pour objet les récepteurs activés par les proliférateurs de peroxysomes (PPAR) comme médiateurs des effets des AG. Toutefois, même si certains effets transcriptionnels des AG passent par leur liaison à ces récepteurs pour les activer, ce mécanisme est loin d'être général. Par ailleurs, il ne s'applique qu'aux effets stimulateurs de la transcription alors que l'action négative des AG ne fait pas intervenir les PPAR.

Le cas de régulation négative le plus documenté est celui de la synthase des AG (FAS), l'enzyme clé de la lipogenèse. Dans ce cas, les AG eux-mêmes, et non un produit issu de leur métabolisme, sont les molécules actives et seuls les AGPI omega- 3 et omega- 6 ont une action. La vitesse de transcription est affectée. II a été démontré que les $\mathrm{AG}$ diminuent la quantité de sterol regulatory element binding protein- $1 c$ (SREBP-1C), un facteur de transcription, réduisant ainsi la transactivation des gènes cibles de ce facteur tels que ceux des enzymes de la lipogenèse [14]. Le mécanisme précis n'est pas encore complètement élucidé, mais il semblerait que les $A G$ exercent une action posttranscriptionnelle conduisant à une diminution de la demi-vie des messagers codant SREBP-1c et de la quantité de cette protéine.

L'activation de la lipogenèse et l'induction de la FAS sont des phénomènes couramment observés lors de la cancérogenèse mammaire et du cancer de la prostate. L'hypothèse la plus probable est que les cellules en prolifération active ont besoin $d^{\prime}$ une synthèse accrue $d^{\prime} A G$ pour les incorporer dans leurs phospholipides membranaires. La FAS est donc une cible de l'état cancéreux. L'existence d'une corrélation positive entre la présence d'une quantité élevée de FAS, de SREBP-1c et le caractère agressif de certains cancers (prostate, sein) a été décrit et la FAS est considérée comme une cible dans ce cas [15]. D'autres exemples de régulation négative imputée à une inhibition par les AG du potentiel de transactivation de gènes cibles par des facteurs de transcription ont été décrits et ont fait l'objet d'articles de revue récents [4, 13]. On peut citer :

- le facteur nucléaire 4 des hépatocytes (HNF-4) qui lie les AG activés en acyl-CoA ; - le récepteur des hormones thyroïdiennes (T3) ;

- le facteur de transcription dénommé «protéine de liaison à l'élément de réponse au glucose » ou ChREBP via lequel les AG à longue et à courte chaîne répriment l'effet du glucose sur le gène codant la pyruvate kinase hépatique ; - le facteur de transcription AP-1 qui répond aux esters de phorbol et dont les effets sont inhibés spécifiquement par les AGPI omega-3 dans les cellules J6B d'épiderme de souris. En revanche, I'acide arachidonique n'a pas d'effet. Ainsi, dans ce cas apparaît clairement une différence d'action des AGPI des deux familles ; - le complexe Myc-Max. Dans une lignée humaine de cancer de l'estomac, les cellules SNU16, les AGPI sont capables d'interagir avec ce complexe, inhibant sa liaison à ses séquences cibles de reconnaissance, les boîtes $E$. c-Myc étant le produit du protooncogène $c-M Y C$, I'hypothèse est que les AGPI s'opposent à l'activité oncogénique de cette protéine.

Enfin, la plupart des études démontrant un effet inhibiteur des AG sur les gènes concernent les AGPI, sensibles à la peroxydation. II a donc été suggéré que les produits de peroxydation peuvent avoir des effets cytotoxiques rendant compte d'un effet négatif. Dans ce cas, la vitamine E devrait s'opposer à l'action des AGPI.

Les PPAR ont été considérés comme les médiateurs des effets transcriptionnels positifs des AGPI. II en existe trois isoformes (PPARa, $\operatorname{PPAR} \beta / \delta$ et PPAR $\gamma$ ) ayant des sélectivités $\mathrm{d}^{\prime}$ expression tissulaire. PPAR $\alpha$ est ainsi fortement exprimé dans le foie alors que PPAR $\gamma$, bien que présent dans beaucoup de tissus, a une expression prépondérante dans le tissu adipeux et les macrophages. Les PPAR forment des hétérodimères avec le récepteur de l'acide rétinoïque 9-cis (RXR) pour se lier à des éléments de réponse dans le promoteur des gènes cibles, les PPRE. De façon notable, les trois isoformes sont aussi capables de lier les AGPI et plus particulièrement le DHA. L'activité de ce récepteur est donc aussi potentiellement altérée par les AG. Les PPRE ont une structure de type direct repeat 1 (DR1). En plus des AG, d'autres ligands naturels des PPARs ont été décrits, tels certaines prostaglandines [16]. Les PPAR semblent impliqués dans le cancer. En effet, l'activation de PPAR $\gamma$ permet de stopper la prolifération de plusieurs cellules tumorales, $d^{\prime}$ 'induire leur différenciation et/ou de les faire entrer les cellules en apoptose [4, 17]. En revanche, I'activation de PPAR $\beta / \delta$ semble plus spécifiquement entraîner une hyperprolifération et favoriser l'apparition du cancer, notam- 
ment dans le cas du côlon. On peut donc considérer que ces deux isoformes de PPAR semblent exercer des effets opposés sur la prolifération bien que certaines études nuancent ce résultat.

L'action anti-inflammatoire des PPAR a été très documentée [16]. PPAR $\alpha$ et PPAR $\gamma$ sont exprimés dans les macrophages dont ils régulent la réponse aux stimuli inflammatoires. Des études ont montré que la production de cytokines pro-inflammatoires (TNF- $\alpha$, IL-6, IL-1 $\beta$ ) par les monocytes est réduite sous l'influence d'activateurs potentiels des PPAR, comme la 15-desoxy PG-J2, mais ces résultats sont controversés [16]. Plusieurs autres études indiquent que les agonistes des PPAR, comme les fibrates, pourraient interférer de façon négative avec la signalisation NF- $\mathrm{KB}$, entraînant l'inhibition de la sécrétion d'interleukines comme l'IL-6.

Bien que beaucoup de zones d'ombre persistent quant à l'importance réelle des PPAR dans la réponse inflammatoire et le cancer, l'effet des AGPI sur la régulation de l'expression de gènes codant des protéines de l'inflammation est assez bien établi. Par exemple, l'EPA et le DHA s'opposent à la stimulation de l'expression de COX-2, IL-1 $\beta$, ou TNF- $\alpha$ dans les explants de cartilage arthritique humain [18]. Par ailleurs, les AGPI peuvent empêcher l'activation de NF-kB par le TNF- $\alpha$, peut-être par une diminution de phosphorylation de l'inhibiteur de NF- $\kappa B$, dénommé IкB $[19,20]$. Dans tous ces cas de figure, les omega-3 sont encore signalés comme anti-inflammatoires.

\section{Le cas particulier de l'acide butyrique}

Le n-butyrate, un AG saturé à 4 carbones est issu de la fermentation des sucres bactériens dans le colon. II est désormais reconnu qu'une perturbation des relations entre microflore bactérienne et épithélium colique peut entraîner I'inflammation des muqueuses et éventuellement la transformation cellulaire conduisant au cancer colique [21]. II est aussi établi que la consommation de fibres peut avoir un rôle protecteur vis-à-vis du cancer du colon. Le n-butyrate pourrait être le médiateur de ces effets protecteurs des fibres, par ses propriétés anti-prolifératives et inductrices de l'apoptose, démontrées dans différents modèles cellulaires de cancer (côlon, prostate, foie, sein). Le mécanisme d'action du butyrate sur l'expression des gènes est très documenté. II s'agit d'un inhibiteur des enzymes qui désacétylent les histones (HDAC). De façon très schématique, l'acétylation des histones est liée à une structure de chromatine ouverte et à l'expression des gènes alors que la désacétylation referme la chromatine et entraîne une répression de la transcription [22]. L'empêchement de la désacétylation des histones par le butyrate est donc favorable à l'expression des gènes. Étant donné que la répression inappropriée de la transcription sous I'effet des HDAC est un mécanisme utilisé par les oncoprotéines pour leur action tumorigène, il devient possible d'expliquer l'effet antitumoral du butyrate par son action inhibitrice de ces enzymes. Plusieurs essais cliniques utilisant cet AG et ses dérivés sont en cours, notamment dans le cancer du côlon.

\section{Acides gras et système immunitaire dans I'inflammation et le cancer}

Les recherches ayant pour objet l'étude de l'effet des AG oméga comme modulateurs de I'inflammation et des réponses immunitaires sont abondantes et il en est de même de la littérature à ce sujet $[1,23]$. Ce chapitre ne se veut donc pas exhaustif mais présente un simple aspect du problème, en liaison avec la lipomobilisation des AG du tissu adipeux. En réponse à une infection virale ou bactérienne, I'organisme met en place une série de réactions immunes. En particulier, les tissus infectés sont colonisés par des macrophages et des lymphocytes qui secrètent diverses cytokines dont, respectivement, le TNF- $\alpha$ et l'interféron gamma (IFN- $\gamma)$. Comme il est signalé ci-avant, le TNF- $\alpha$ ainsi que IL-1 et IL- 6 sont proinflammatoires. De façon très intéressante, le TNF- $\alpha$ et I'IFN- $\gamma$ sont fortement lipolytiques [24]. On peut donc imaginer que les macrophages et/ou les lymphocytes T colonisant les tissus sécrètent ces médiateurs, en réponse à une agression bactérienne et/ou virale, ce qui aura pour conséquence une mobilisation des AG du tissu adipeux avoisinant. Cette hypothèse a été récemment bien débattue, notamment dans le cadre de la lipolyse du tissu adipeux au voisinage des ganglions lymphatiques lors d'une inflammation locale [25]. Les AG peuvent alors servir de substrat énergétique, par exemple pour la maturation et la prolifération lymphocytaire, mais aussi de molécules de signalisation. En effet, la lipomobilisation des AC à partir des triglycérides du tissu adipeux présente une certaine sélectivité [26]. Par exemple, les AGPI oméga de réserve sont plus rapidement libérés que les autres AG. En conséquence, la qualité des AG stockés dans le tissu adipeux, influencée fortement par l'alimentation, peut avoir des répercussions importantes sur la réponse inflammatoire de l'organisme et, potentiellement, sur le dérèglement de la prolifération cellulaire en liaison avec cet état physiopathologique.

\section{Conclusion}

La reconnaissance des actions proinflammatoire des AGPI omega- 6 et antiinflammatoire des AGPI omega- 3 a conduit les cliniciens à postuler qu'un régime enrichi en omega-3, proportionnellement aux omega-6, pouvait combattre l'inflammation chez des patients atteints de diverses affections telles que la maladie de Crohn, le lupus, l'asthme, le psoriasis etc. Des essais thérapeutiques ont été effectués, montrant le plus souvent un effet de réduction des médiateurs inflammatoires sanguins [6]. Toutefois, hormis la démonstration d'une action bénéfique dans le cas de l'arthrite rhumatoïde, les preuves manquent dans la plupart des autres cas et d'autres essais sont nécessaires. En revanche, si les mécanismes antiinflammatoires des omega- 3 sont encore très partiellement connus, l'utilisation de ces AG en clinique semble prometteuse.

\section{RÉFÉRENCES}

1. PHILPOTT M, FERGUSON LR. Immunonutrition and cancer. Mutat Res 2004 ; 551 : 29-42.

2. GRETEN FR, ECKMANN L, GRETEN TF, et al. IKK beta links inflammation and tumorigenesis in a mouse model of colitis-associated cancer. Cell $2004 ; 6$ : 285-96.

3. BOUGNOUX P, MENANTEAU J. Acides gras alimentaires et cancérogenèse : données expérimentales in vivo. Bull Cancer 2005 ; 92 : 68596.

4. BARDON S, BENELLI C, BERNARD-GALLON D, et al. Acides gras alimentaires et cancer : mécanismes d'action cellulaire et moléculaire. Bull Cancer $2005 ; 92: 697-707$.

5. ALAM SQ, REN YF, ALAM BS. [3H]forskolinand $[3 \mathrm{H}]$ dihydroalprenolol-binding sites and adenylate cyclase activity in heart of rats fed diets containing differnt oils. Lipids $1988 ; 23$ : 207-13.

6. CALDER P. Polyunsaturated fatty acids and inflammation. Prost Leuk Ess 2006 ; 75 : 197 202.

7. KARGMAN SL, O'NEILL GP, VICKERS PJ, EVANS JF, MANCINI JA, JOTHY S. Expression prostaglandin $\mathrm{G} / \mathrm{H}$ synthase- 1 and -2 protein in human colon cancer. Cancer Res 1995 ; 55 : 2556-9.

8. PRESCOTT SM, FITZPATRICK FA. Cyclooxygenase-2 and carcinogenesis. Biochim Biophys Acta $2000 ; 1470$ : M69-M78.

9. KERN MA, SCHONEWEISS MM, SAHI D, et al. Cyclooxygenase-2 inhibitors suppress the growth of human hepatocellular carcinoma implants in nude mice. Carcinogenesis 2004 ; $7: 1193-9$. 
10. TSUJII M, DUBOIS RN. Alteration in cellular adhesion and apoptosis in epithelial cells overexpressing prostaglandin endoperoxide synthse 2. Cell $1995 ; 83: 493-501$.

11. BAGGA D, WANG L, FARIAS-EISNER R, GLASPY JA, REDDY ST. Differential effects of prostaglandin derived from omega- 6 and omega- 3 polyunsaturated fatty acids on COX-2 expression and IL-6 secretion. Proc Natl Acad Sci USA $2003 ; 100: 1751-6$.

12. FRANK J, POMPELLA A, BIESALSKI HK. Histochemical visualization of oxidant stress. Free Radic Biol Med 2000 ; 29 : 1096-105.

13. DUPLUS E, FOREST C. Is there a single mechanism for fatty acid regulation of gene transcription? Biochem Pharmacol 2002 ; 64 : 893-900.

14. WORGALL T, STURLEY SL, SEO T, OSBORNE TF, DECKELBAUM RJ. Polyunsaturated fatty acids decrease expression of promoters with sterol regulatory elements by decreasing levels of mature sterol regulatory element-binding protein. J Biol Chem 1998 ; 273 : 25537-40.
15. MENENDEZ JA, LUPU R. Oncogenic properties of the endogenous fatty acid metabolims: molecular pathology of fatty acid synthase in cancer cells. Curr Opi Clin Nutr Metab Care $2006 ; 9$ : 346-57.

16. CHINETTI G, FRUCHART JC, STAELS B. Peroxisome proliferator-activated receptors : new targets for the pharmacological modulation of macrophage gene expression and function. Curr Opin Lipidol 2003 ; 5 : 459-68.

17. BASTIE C. PPAR delta and PPAR gamma : roles in fatty acid signalling. Implication in tumorigenesis. Bull Cancer 2002 ; 89 : 23-8.

18. CURTIS C, REES SG, LITTLE CB, et al. Pathologic indicators of degradation and inflammation in human osteoarthritic cartilage are abrogated by exposure to n-3 fatty acids. Arth Rheum $2002 ; 46: 1544-53$.

19. NOVAK TE, BABCOCKTA, JHO DH, HELTON WS, ESPAT NJ. NF- $\kappa B$ inhibition by $\omega-3$ fatty acids modulate LPS-stimulated macrophage TNF- $\alpha$ transcription. Am / Physiol 2003 ; 284 . L84-L89.
20. ZHAO Y, JOSHI-BARVE S, BARVE S, CHEN LH. Eicosapentaenoic acid prevents LPS-induced TNF-a expression by preventing NF-kB activation. J Am Coll Nutr 2004 ; 23 : 71-8.

21. SCHEPPACH W, WEILER F. The butyrate story: old wine new bottles? Curr Opin Clin Nutr Metab Care 2004 ; 7 : 563-7.

22. MONNERET C. Histone deacetylase inhibitors. Eur J Med Chem 2005 ; 40 : 1-13.

23. FRITSCHE K. Fatty acids as modulators of the immune response. Annu Rev Nutr 2006; 26 : 45-73.

24. KHOVIDHUNKIT W, KIM MS, MEMON RA, SHIGENAGA JK, MOSER AH, FEINGOLD KR, GRUNFELD C. Effects of infection and inflammation on lipid and lipoprotein metabolism: mechanisms and consequences to the host. J Lipid Res 2004 ; 45 : 1169-96.

25. POND CM. Adipose tissue and the immune system. Prost Leuk Ess 2005 ; 73 : 17-30.

26. RACLOT T. Selective mobilization of fatty acids from adipose tissue triacylglycerols. Prog Lipid Res $2003 ; 42$ : 257-88. 